\title{
Towards a resolution of the personality triad: Persons, situations, and behaviors ${ }^{\text {th }}$
}

\author{
David C. Funder* \\ Department of Psychology, University of California, Riverside, CA 92521, USA
}

Available online 5 October 2005

\begin{abstract}
The issue of the variant vs. invariant in personality often arises in different forms of the "personsituation" debate, which is based on a false dichotomy between the personal and situational determination of behavior. Previously reported data are summarized that demonstrate how behavior can vary as a function of subtle situational changes while individual consistency is maintained. Further discussion considers the personal source of behavioral invariance, the situational source of behavioral variation, the person-situation interaction, the nature of behavior, and the "personality triad" of persons, situations, and behaviors, in which each element is understood and predicted in terms of the other two. An important goal for future research is further development of theories and methods for conceptualizing and measuring the functional aspects of situations and of behaviors. One reason for the persistence of the person situation debate may be that it serves as a proxy for a deeper, implicit debate over values such as equality vs. individuality, determinism vs. free will, and flexibility vs. consistency. However, these value dichotomies may be as false as the person-situation debate that they implicitly drive.

(c) 2005 Elsevier Inc. All rights reserved.
\end{abstract}

Keywords: Personality; Persons; Situations; Behaviors; Person-situation interactions

\footnotetext{
Data gathering for Funder and Colvin (1991) and Furr and Funder (2004), summarized in this paper, was supported by NIMH Grant R01-MH42427 to David Funder.

* Fax: +1951 8273985.

E-mail address: funder@ucr.edu.
} 


\section{Introduction}

The issue of the variant vs. invariant in personality translates into a concern over whether the behavior of a typical person is consistent (invariant) enough across time and situations to be usefully attributed to individual characteristics. The alternative possibility is that behavior is so inconsistent (variable) that only the situation matters. For the past several decades this fundamental issue has recurred in various forms and under various labels, the most common of which is the "person situation debate" (Kenrick \& Funder, 1988).

The persistence of this debate into the 21 st century is something of a mystery. Since at least the 1930s, deep thinkers as diverse as Allport (1937) and Lewin (1951) have argued that invidious comparisons miss the point because behavior is a function of an interaction between the person and the situation. By the 1980s this recognition had deteriorated into a truism. Nowadays, everybody is an interactionist. Still, the argument persists. In the modern personality literature, it is not unusual for the obligatory recital of interactionist clichés to be immediately followed by some sort of implicit but clear revelation of which variable the writer favors, usually the situation. Sometimes this preference is expressed subtly, such as via a graceful paean to the flexible adaptivity of human nature. Other times expression is blatant, as in citations of the "fundamental attribution error" (FAE), the putative tendency to think that aspects of the person importantly contribute to behavior when really the situation is all that matters. Gentler expositions of the FAE suggest that people emphasize the causal power of the person too much, and the power of the situation not enough. Either way, to treat the relative contributions of person and situation to behavior as a zero-sum game in this manner is to demonstrate the exact misunderstanding that the interactionist consensus had supposedly risen above.

Precisely because the interactionist consensus has become a truism, it is rarely if ever closely examined. The purpose of the present article is to seek to begin such a re-examination. I shall reframe the person-situation interaction in the determination of behavior in terms of a "personality triad" of persons, situations, and behaviors, in which not only do the first two elements determine the third, but each of the other pairs likewise determines the remainder. I shall try to work through some of implications of this point of view, suggest directions, as always, for future research, and finally end on a philosophical note that takes me far, far away from any data.

\section{The situation and the person}

The person-situation debate has generated no shortage of arguments, but directly relevant data remain scarce. To yield relevant data, the behavior of a sample of subjects must be directly measured in more than one situation, so that their consistency can be assessed. Direct behavioral measurement is difficult and expensive and accordingly has been rare in personality research (Funder, 2001). Repeated behavioral measurement has been even rarer. The very recent literature includes signs that this situation may be starting to change (e.g., Mehl \& Pennebaker, 2003; Wolf, Borkenau, Angleitner, Riemann, \& Spinath, 2004), which is good, because when behavior is directly measured on more than one occasion, illuminating analyses become possible.

To illustrate, consider a simple study Randy Colvin and I published a few years ago (Funder \& Colvin, 1991). One hundred and forty undergraduate subjects were observed in 
two different experimental situations. In the first, two undergraduates of the opposite sex who had never met before were shown into a small room containing little except a couch and a video camera. The experimenter told them that they could "talk about whatever you like," said he would be back in a few minutes, activated the camera (in plain view), and left. The second situation occurred a few weeks later, and was exactly like the first, except that each subject was paired with a different opposite-sex partner, and both of them were there for the second time.

The usual behavior in this situation amounted to a kind of getting-acquainted conversation, but within that limit still varied widely across individuals. To capture this variation, we employed an early version of the Riverside Behavioral Q-sort (RBQ; Funder, Furr, \& Colvin, 2000), which included 62 items describing overt aspects of social interaction. Trained research assistants viewed each videotape, and then sorted these items into a 9-step distribution ranging from very characteristic to very uncharacteristic of the behavior of the individual who was observed. Four research assistants viewed each tape and their ratings were averaged; nobody rated or saw a given subject more than once.

\subsection{The situation}

In essence, this is a simple repeated-measures experiment, with 62 dependent variables. Data analysis is equally simple. For each of the items, a repeated-measures $t$ test can reveal whether the behavior it describes varied significantly between the two situations. Some results are shown in Table 1 (for the full results, see the original article). At the first session, subjects' behavior was rated as relatively awkward, tense, disinterested, distant, insecure, and fearful. But by the second session, behavior was observed to have become more relaxed, socially skilled, interesting, expressive, fluent, and all-around enjoyable. These results are not difficult to explain. At the second session, subjects were

Table 1

The effect of the situation: mean differences in behavior between the first and second experimental session

\begin{tabular}{llll}
\hline Behavioral Q-sort item & Session 1 & Session 2 & \\
\hline Items higher at session 1 & & 3.51 & 4.96 \\
Talks at rather than with partner & 3.98 & 3.60 & 4.50 \\
Exhibits an awkward interpersonal style & 4.19 & 4.66 & 3.76 \\
Shows physical signs of tension or anxiety & 5.19 & 3.55 & 3.33 \\
Shows lack of interest in the interaction & 3.98 & 4.40 & 2.97 \\
Keeps partner at a distance & 4.81 & 4.49 & 2.93 \\
Expresses insecurity or sensitivity & 4.77 & 3.64 & 2.85 \\
Behaves in a fearful or timid manner & 3.98 & & 4.65 \\
Items higher at session 2 & & 6.46 & 3.98 \\
Exhibits social skills & 5.94 & 6.13 & 2.79 \\
Appears to be relaxed and comfortable & 5.56 & 6.08 & 2.68 \\
Says or does interesting things & 5.78 & 5.42 & \\
Is expressive in face, voice or gestures & 5.11 & & \\
\hline
\end{tabular}

Note. $N=140, d f=138$. All differences are significant at $p<.01$. Table is adapted and abbreviated from Funder, D.C., \& Colvin, C.R. (1991). Explorations in behavioral consistency: Properties of persons, situations and behaviors. Journal of Personality and Social Psychology, 52, p. 783, published by the American Psychological Association. Reprinted by permission. 
much more at ease. They had been in the laboratory situation before, they had even met the research assistant, and the environment had transformed itself from one that was strange and unpredictable to one in which they visibly felt and acted much more comfortable. Overall, 20 of the 62 behavioral items changed between the two sessions (at the $p<.05$ level, at which about four would be expected by chance), all of which are consistent with this interpretation.

This is exactly the kind of evidence so often used to demonstrate the power of the situation, and rightfully so. While the two situations may at first glance seem only slightly different, it is clear that on a psychological level the difference between being in an experiment for the second time as opposed to the first time can be powerfully important, with major, observable and even dramatic effects on behavior. In the psychological literature we have often read phrases to the effect that seemingly small changes in situations can have major psychological importance, and here we see a sterling example.

\subsection{The person}

But what about behavioral consistency? Effects like these, and phrases such as just mentioned, are often taken to imply that the consistency of behavior across these situations, and therefore the influence of personality on behavior in them, is - must be-low. However, the influence of personality is reflected in individual differences in behavior and that requires a separate analysis.

The analysis is again simple. For each of the 62 behavioral items, one calculates a Pearson correlation between individuals' behavior at Time 1 and their behavior at Time 2 . Some of the results are reported in Table 2. As can be seen, the consistencies are numerous and impressive. Some of the cross-situational correlations exceed .60. Our subjects were quite consistent in the relative degree to which they spoke loudly, acted timidly, laughed, smiled, and were expressive, unexpressive, awkward, and enthusiastic. Overall, 37 of the 62 correlations calculated were significant at $p<.001$, of which 34 were higher than .30 (once touted as the "personality coefficient") and 25 were higher than .40 (only this last group is shown in Table 2; for the complete results see Funder \& Colvin, 1991).

But wait. What is so impressive about all this consistency? Were not these situations pretty similar, after all? No. If the preceding analysis of behavioral change made no other point, it surely made that one. These situations, seemingly similar on a superficial level, turned out to be psychologically very different, and consequentially so. People felt differently between them and changed their behavior accordingly. At the same time, they maintained their individuality. The most fearful people in the first session were still the most fearful-compared to their peers - in the second situation, the most expressive in the first situation were still the most expressive in the second situation, and so forth.

\subsection{Situations and persons}

The point of this demonstration is not that it is possible to demonstrate impressive degrees of cross-situational consistency to behavior (although admittedly it does make that point rather well), but that behavioral change and individual consistency are orthogonal phenomena. Indeed, the same data here offer three lessons. First, they underline the power 
Table 2

The effect of the person: cross-situational consistency correlations between the first and second experimental session

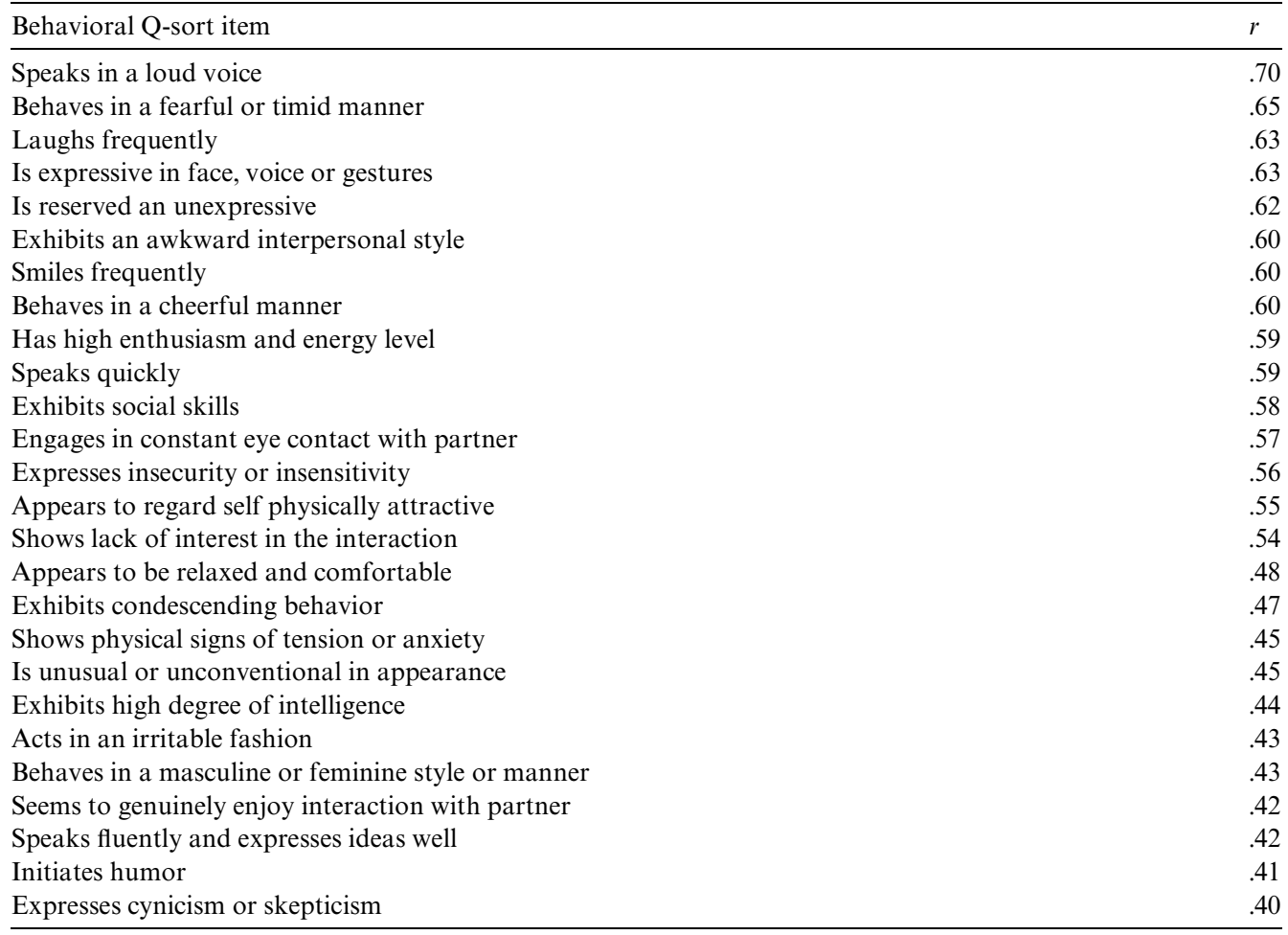

Note. $N=140$. All correlations are significant at $p<.001$ (two tailed). Table is adapted and abbreviated from Funder, D.C., \& Colvin, C.R. (1991). Explorations in behavioral consistency: Properties of persons, situations and behaviors. Journal of Personality and Social Psychology, 52, p. 780, published by the American Psychological Association. Reprinted by permission.

of the situation - including seemingly minor aspects of situations - to change behavior in major and important ways. Second, they demonstrate the impressive stability of individual differences in behavior across situations. Third, they demonstrate the independence of behavioral change and consistency, at the item level as well as at the aggregate level. In a further analysis, we correlated the degree to which each of the 62 items changed (in mean level) across the two situations with its cross-situational consistency correlation. The correlation was -.01 .

The important contrast revealed in these data, therefore, is not the traditional one between behavioral variance and invariance, but between examining behavior at the level of the sample as opposed to the level of the individual. At the sample level, many behaviors changed across the two situations - some did not. At the individual level, many behaviors maintained consistency between the two situations; a few did not. But these were not necessarily the same behaviors. The -.01 correlation just mentioned demonstrates that the properties of a situation that make it susceptible to mean level change across situations are unrelated to those that make it likely to maintain individual differences across situations. Consider a single individual who was lucky enough to be recruited for our study. Like most 
of his peers, he was probably much less nervous at the second session than he was at the first session. At the same time, if he was more nervous than most participants at the first session, he was probably still also more nervous than most participants at the second session.

The moral of this story is that the variant and invariant in personality are not competing phenomena, though they often are discussed as if they were. Except in the rare cases where individual differences in behavior are utterly obliterated (are there any such?), one does not imply anything about the other. This ought to be a basic methodological point understood by everyone, certainly everyone with a Ph.D. in personality or social psychology. But I fear it is not. One reason for this fear is that I still come across articles that trumpet the powerful effect of situations on behavior as if they somehow undermine the importance of individual differences in personality. (For some reason, I do not see the reverse point made very often.) Until this stops, demonstrations such as the one just summarized will continue to be necessary (see also Fleeson, 2001, 2004).

If the point of this demonstration is grasped, then the future of personality psychology can (finally) become more interesting. In the remainder of this paper I shall briefly consider five aspects of the field's agenda: (1) the source of behavioral invariance, (2) the source of behavioral variation, (3) the person-situation interaction, (4) the nature of behavior, and (5) the personality triad of persons, situations, and behaviors.

\section{The source of behavioral invariance}

A definitive task for personality psychology is to further explicate the source of the behavioral invariance or behavioral consistency that is repeatedly found, irrespective of the situation. The first step in this enterprise is to assimilate the patterns of behavioral consistency to broader traits that subsume and begin to explain them. For example, it is not difficult to assimilate many of the consistent behaviors in Table 2 to a trait we could reasonably call extraversion, which implies that people are consistent across these two situations because they possess varying degrees of this trait and express it in both contexts. The next step is to seek the source of the trait, with the usual suspects being genes, the environment and, of course, their interaction. (Indeed, the "nature-nurture" controversy parallels the "person-situation" controversy in many ways, including its putative resolution (interactionism) and its conceptual pitfalls.)

For example, to seek the source of the consistencies illustrated in Table 2, one might investigate the child-rearing tactics that produce extraverted children, the temperamental bases of extraversion, and the way these two elements interact. This is the traditional and honorable agenda of personality psychology, and much has been accomplished along these lines. Still, much remains to be done, especially concerning the way personality is manifested in behavior (Funder, 2001).

A key aspect of this research tradition is that it ignores behavioral variation due to the situation. Critics of personality psychology often lament this fact. But if one is interested in the stable characteristics of a person that contribute to his or her behavior regardless of the situation he or she inhabits, this is really the only way to proceed. Once a researcher has assessed the ways in which people consistently act differently from each other, it becomes possible to seek the sources of these individual differences. It can be useful to ignore the situation to highlight the ways in which people are different from each other. 


\section{The source of behavioral variation}

The situation is important too, of course. But it is difficult to pin down just how situations are important, in part because of the common but unilluminating practice of assigning "the situation" responsibility for all the behavioral variance not accounted for by a particular personality trait, without specifying what aspects of the situation are psychologically essential (Ahadi \& Diener, 1989; Funder \& Ozer, 1983). There is a good deal of confusion concerning how situations should be conceptualized.

On the one hand, it has frequently been argued that the psychologically important aspects of situations lie in the eye of the beholder. As Gordon Allport wrote, "similarity is personal" (1937, p. 283). Bem and Allen (1974) argued that "the classification of situations...will have to be in terms of the individual's phenomenology, not the investigator's" (1974, p. 518), and Mischel has observed that "any given, objective stimulus condition may have a variety of effects, depending on how the individual construes and transforms it" (1977, p. 253). These comments might seem to imply that the only valid way to assess a situation would be ask each individual in it for his or her possibly idiosyncratic perceptions of its salient aspects.

On the other hand, there are reasons to wish for a more objective conceptualization that does not require a situation to be redefined a new for each individual who encounters it. Allport also acknowledged that "some basic modes of adjustment...from individual to individual are approximately the same" (1937, p. 298), by which he meant that while each individual's view of reality is ultimately unique there is enough overlap from one person to another to allow meaningful and useful classification of stimuli. Moreover, people are not always aware of the aspects of situations that drive their behavior; behavioral as well as psychoanalytic psychotherapy begins with an attempt to identify the aspects of the client's life that importantly affect his or her behavior, and often one problem turns out to be that the client is not initially aware of what these are. A further consideration is that an objective conceptualization of situations raises the possibility of experimental or therapeutic manipulation. If the behaviorally effective aspects of a situation can be identified, then the situation can be changed to affect behavior in the desired way, at least on average.

The most important reason for psychologists to seek objective ways of conceptualizing situations is that to define a situation in terms of the perceptions of the persons in it runs the risk of circularity. If a situation is defined as "hostility evoking" for a particular person because it makes this particular person feel "hostile," there is no way to separate out the degree to which the person is dispositionally hostile all the time from the degree to which the situation would deeply aggravate anybody. A related concern is that the "eye of the beholder" conceptualization of situations is suspiciously similar to the post-modern, deconstructionist philosophy that has influenced literary criticism and much of the rest of the humanities, a philosophy that is interesting but also, it could be argued, fundamentally anti-scientific.

The distinction between personal and objective definitions of situations was addressed in a pair of studies recently published by Mike Furr and myself (Furr \& Funder, 2004). Study I was based on the same Harvard data set that was the basis of the demonstration summarized earlier. After the end of their second experimental session, the participants in that study were asked to rate, on a simple Likert scale, "how similar" they found the two situations to be. We correlated these ratings with a variable-centered and a person-centered measure of each individual's behavioral consistency across the two situations, and in both 
cases found a positive correlation ( $r=.28$ and $r=.23$, respectively, $p<.01$ for both). The more similar a person rated the two situations, the more consistently he or she behaved across them. This finding confirms that the "same" two situations can differ in how similar they seem to different participants, and the subjective similarity in turn is behaviorally consequential.

Study II looked at objective similarity. In this study, the behavior of 180 undergraduates at the University of California, Riverside was videotaped in each of 6 situations with two different partners as they performed three different tasks. Thus, 9 "similar" pairs of these situations objectively shared one element (either the partner or task), and 6 "dissimilar" pairs shared neither element. Again, the behavioral consistency was assessed using a variable-centered and person-centered measure, and in both cases objective situational similarity had a strong effect on behavioral consistency-95\% of the individual participants and $98 \%$ of the behavioral variables assessed were more consistent across the similar than across the dissimilar situations.

These findings are good news. People are more consistent across "more similar" situations, compared to dissimilar situations, no matter whether the assessment of consistency is variable centered or person centered, and whether situational similarity is defined subjectively or objectively. These findings reassure us that participants have some degree of access to and ability to rate the behaviorally important aspects of the situations they find themselves in. They also suggest that the psychologically salient aspects of situations enter the mind of the beholder via his or her contact with objective reality. Most people do not hallucinate. Their subjective representation of reality, which is what matters at a psychological level, is typically closely related to the objective nature of reality. This is a good thing, because it means that objective features of situations, such as the identity of the participants or the nature of the task, are reasonable to include in psychological analysis. The objective basis of subjectivity also is what makes possible phenomena such as social interaction, civilization, and survival. While it is true that one's behavior with respect to a cliff depends upon one's subjective representation of it, jumping off of it is a bad idea, objectively speaking.

The particular variables that objectively defined the situations in the Furr study were ad hoc and used only because they were available - surely there is more to a situation than the identity of one's partner and the task one is assigned. An important future direction for personality psychology, therefore, is to begin to formulate the variables that psychologically characterize situations. Most of the few, early attempts in the current literature seem to focus on lexical analyses (e.g., Van Heck, Perugini, Caprara, \& Froeger, 1994; Yang, Read, \& Miller, in press; who used Chinese idioms), but ultimately the test of the adequacy of a set of situational variables will be the degree to which they can predict and are useful for explaining behavior (see, e.g., Kelly et al., 2003; Ten Berge \& De Raad, 2002).

\section{The person-situation interaction}

As was mentioned earlier, the claim that behavior is best conceptualized as the result of an interaction between the person and the situation has attained the status of a truism. There is something ironic, therefore, in the fact that reliable and replicable interactions have proven difficult to find (Chaplin, 1991). Indeed, when one looks again at the robust main effects of both the situation and the person in Tables 1 and 2, it is possible to wonder how much stable and meaningful behavioral variance is really left for the per- 
son-situation interaction to account for. Every practicing psychological researcher knows that robust main effects are much easier to find than are replicable two-way (not to mention higher-order) interactions, and the literature as a whole contains remarkably few. This observation does not mean that person-situation interactions are not important, but it does caution us that we should not expect them to be ubiquitously strong, easy to find, or replicable.

\subsection{If-then patterns}

One prominent way to conceptualize the person-situation interactions is in terms of what Mischel and Shoda (1995) call "if-then" patterns of behavioral variability, as is illustrated in one of their figures reproduced in Fig. 1. The Figure illustrates that while Person A and Person B have the same average level of Behavior X, the pattern of the expression of this behavior across situations is different for the two individuals. For example, Behavior $\mathrm{X}$ is equally likely for both persons in Situation 3, more likely for Person B than for Person A in Situation 4, and more likely for Person A than for Person B in Situation 8. Mischel and Shoda suggest that this is how psychologists should conceptualize not just person-situation interactions but individual differences in general. Their approach enjoys an important advantage over the conventional method of regarding Person A and Person B as equivalent in their average manifestation of Behavior X, by showing that this similarity potentially hides important differences in the patterns of their behavior.

This conceptualization also raises several questions. One set of questions is empirical, and arises from the fact that Person A, Person B, and Behavior X, and their elegant patterns, are all hypothetical. While a few empirical demonstrations have been reported recently (e.g., Wright, 2001), it is still not clear how often this kind of within-person behavioral variability will be found, how often it will be stable, and how often it will be meaningful. The difficulties already mentioned that psychologists have had in finding strong and replicable person-situation interactions suggest that whatever empirical success the if-then conceptualization achieves, it will not be won easily.

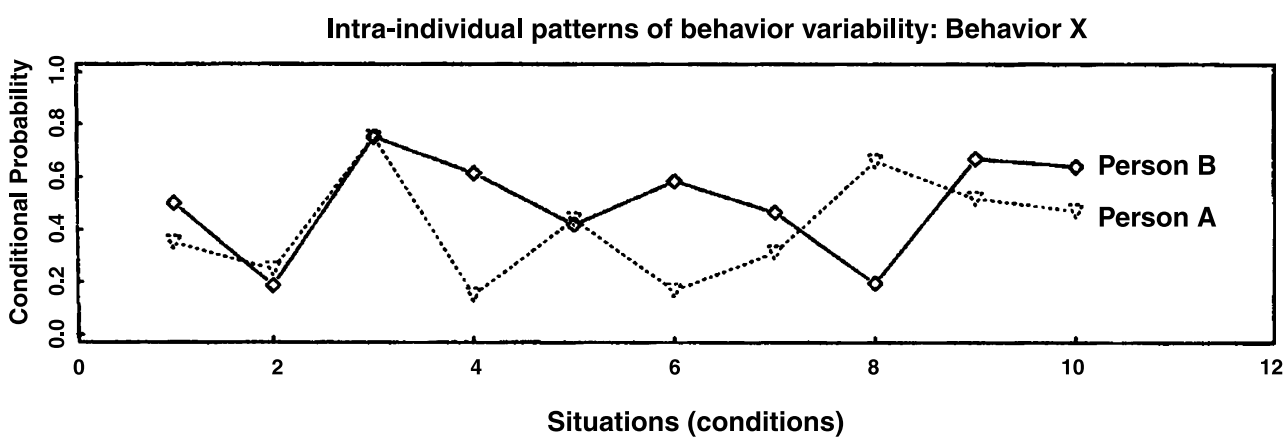

Fig. 1. Illustration of Mischel and Shoda's "if-then" conceptualization of individual differences in behavior. The chart shows the conditional probability of Behavior X for persons A and B across Situations 1-12. From Mischel, W., \& Shoda, Y. (1995). A cognitive-affective system theory of personality: Reconceptualizing situations, dispositions, dynamics, and invariance in personality structure. Psychological Review, 201, p. 247, published by the American Psychological Association. Reprinted by permission. 
Two further questions are theoretical. First, are the if-then patterns such as shown in Fig. 1 completely idiographic? If so, then personality assessment would seem to demand a different set of if-then charts for every possible behavior, for every person on earth-a daunting prospect. Second, what is the source of these if-then patterns? Classic behaviorism would suggest that each individual's unique pattern of behavior is a function of his or her unique learning history. If that is the case, then the if-then conceptualization of personality amounts to a restatement of some of the more orthodox teachings of B.F. Skinner. More reasonably, it seems likely that "basic modes of adjustment...from individual to individual are approximately the same" (Allport), which implies that a relatively small number of if-then patterns per behavior may be sufficient to account for most individuals. For example, if at a party then most people try to have fun, whereas if at a funeral then most people are inclined to be serious. To the extent a pattern like this holds for all individuals, the approach reduces to an analysis of the main effects of situations. To the extent a pattern like this holds for all individuals but individuals still vary on their mean levels of behavior, then the if-then approach becomes equivalent to an analysis of traits. And to the extent that a limited number of person-situation patterns is sufficient to account for the behavior of most individuals, then the approach amounts to a revival of the type approach to personality - each type being characterized by its own if-then behavioral pattern.

\subsection{Template matching}

A different way to conceptualize the person-situation interaction is to conceptualize situations in terms of the behaviors that different kinds of people perform in them (Bem \& Funder, 1978). For example, consider two undergraduate institutions, UC Berkeley and Harvey Mudd College. The kind of student likely to succeed at Berkeley is assertive, sociable, and self-motivated. A student who is less assertive, less socially engaged and who needs a more constant diet of external support and guidance is more likely to thrive at Harvey Mudd. Notice how nothing has yet been said about the relative enrollment, social atmosphere, or amount of faculty guidance provided at these two institutions, but already a clear view of their differences has begun to emerge. Bem and Funder called this conceptualization of situations "template matching," in which templates describing the persons expected to have particular behavioral outcomes are matched with particular situations. An empirical example was their demonstration study concerning delay of gratification, in which the children who waited longest for a preferred reward (raisins vs. pretzels) were independently characterized not as possessed of iron will and consummate self-control, but rather as being sweet, obedient, cooperative and not particularly intelligent. This finding implied that Bem and Funder's version of this experimental paradigm tapped into a tendency to cooperate with experimenter's wishes more than it revealed mechanisms underlying self-control.

The next step that is required both for Mischel's "if-then" and Bem and Funder's template-matching approach to person-situation interactions is a method for the description of the psychologically important aspects of situations. The hypothetical situations labeled 1-10 in Fig. 1, as well as the delay of gratification situation and others studied by Bem and Funder, will both yield more understanding of the dynamics underlying behavior when psychology has developed a way to conceptualize and measure the aspects of them that are psychologically relevant. As was mentioned earlier, this enterprise is barely begun. 


\section{The nature of behavior}

If situations are under-studied, the matter is even worse when it comes to behaviors. When, as in Fig. 1, a psychologist charts the expression of Behavior X, does it matter what kind of behavior $\mathrm{X}$ is? If so, how can we think about differences between different kinds of behaviors? Only a very few attempts to begin to answer this question have been ventured. Both B.F. Skinner (1938) and McClelland (1984)—psychologists who otherwise were about as different as can be imagined-argued that it was important to differentiate between "operant" and "respondent" behaviors. Briefly, operant behaviors are those that are expressed spontaneously and are in that sense "emitted" by the organism, whereas respondent behaviors are reactions to particular stimuli and are in that sense "elicited" by the environment. It would seem to follow, therefore, that individuals' operant behaviors should be more consistent across situations than are their respondent behaviors, a prediction that was confirmed in a study by Funder and Colvin (1991). More recently, the study cited earlier by Mike Furr and myself (Furr \& Funder, 2004) gathered ratings of behaviors included in the Riverside Behavioral Q-set (Funder et al., 2000) as to the degree to which they were "automatic" as opposed to "controlled." Automatic behaviors included such behaviors as laughing and acting in an animated or expressive fashion. Controlled behaviors included offering advice and expressing criticism. Over all the behaviors assessed, ratings of the degree to which a behavior was automatic as opposed to controlled correlated with cross-situational consistency with an $r=.50$.

While much more remains to be done, the general lesson is clear. Not all behaviors are alike. Personality psychology needs to move beyond analyses of "Behavior X," because Behavior Y might be very different. Some behaviors are more consistent than others, and no doubt they differ in other important ways as well.

\section{The personality triad}

At the core of everything discussed in this article, and perhaps also at the core of personality psychology itself, lies the personality triad, which consists of (1) persons, (2) situations, and (3) behaviors (Funder, 2001, 2004; see also Bem, 1983). A useful way to conceptualize each of these three fundamental elements to personality might be in terms of the other two. A person, for example, can be thought of as the sum total of all of his or her behaviors in all the real and potential situations of his or her life. This is not very different from Mischel's "if-then" conceptualization of personality. In a similar way, a psychological situation can be thought of in terms of the kinds of people who would be expected to perform specified behaviors in it. For example, as we have seen, a college environment could be characterized by the kinds of students who are most likely to succeed and fail, and an experimental delay of gratification situation can be characterized by the kinds of children likely to delay the longest. This is parallel to Bem and Funder's template-matching approach. Finally, and closest to the classic Lewinian conception of interactions, a fruitful way to conceptualize the psychological nature of a behavior might be in terms of situations in which different kinds of people perform it. For example, it might become useful to think of behaviors such as aggression or altuism in terms of the person-situation combinations under which their expression becomes most likely, and such an analysis might illuminate the psychological dynamics that underlie them. 
The Personality Triad

\section{Situation $=$ Behavior $x$ Person \\ Person $=$ Situation $\times$ Behavior \\ Behavior $=$ Person $\times$ Situation}

Fig. 2. Each of the three elements of the personality triad ought to be predictable and explainable in on the basis of the other two.

A second and related implication of the personality triad is that in a fully developed psychological science each of these three terms ought to be derivable from the other two (Fig. 2). For example, if one knew and understood everything about a person and about the situation he or she is in, it ought to be possible to predict what he or she will do (again, this is the classic Lewinian position). By the same token, if one knew everything about a behavior and about a situation, it ought to be possible to predict the kind of person who would act that way under those circumstances. Finally, if one knew everything about a person and the behavior he or she is performing, it ought to be possible to say something about the situation he or she is in.

This analysis reminds us of how persons, situations and behaviors are tightly intertwined. It can be useful to average an individual's behavior across situations to highlight how he or she is different from another individual. It can be useful to average across the behaviors of a group of individuals to highlight how behavior in that situation is different from behavior in another situation. And, it can be useful to cross those two kinds of variables, treating them as independent, to illuminate one form of person-situation interaction. But these analyses, useful as they are, can be potentially misleading if they lead us to forget the mutual dependence of these variables. A situation without people in it has no psychological meaning at all. Every behavior has to be done by somebody, somewhere. A person cannot exist outside of a situation, and a person who has ceased to emit behavior is dead.

\section{Conclusion}

The present paper has argued that personality psychology still has much to do to understand the source and dynamics of person variables, to develop the conceptualization and measurement of situations, and to begin serious consideration of the diverse nature of what has traditionally been monolithically referred to as "behavior." At the same time, it is abundantly clear from every analysis of theory and data that the traditional dichotomy between the person and the situation, in which one gains power only as the other loses, is a false dichotomy and therefore that the person-situation debate is and always was a false debate. So why does the debate persist? Why, for example, do psychologists continue to speak naively of a "fundamental attribution error" that derives directly from a dichotomy that they know, or ought to know, to be false?

Discussions of this putative error and other persistent manifestations of person-situation dichotomization make so little overt sense that one is led to suspect that deeper values and even ideologies may be at stake. Indeed, it is not difficult to think of moral and political values that a situationist outlook might serve, beginning with a basic belief in human 
equality, and ranging further to include beliefs that one enjoys free will only when one can cast off of the shackles of selfhood and invent oneself anew in every situation one encounters, and even ideologies of victimization in which nothing anybody does is her or her fault because behavior is really caused by society, the media, or parental mistakes. The other, person side, begins with an assumption that "one size fits all" is not an appropriate framework for understanding human nature. A person-centered approach might also be seen as favoring values such as a belief that free will resides in the capacity to be true to oneself regardless of the situation one finds oneself in, and that an important purpose in life is to develop a consistent self that seeks to take control of one's own destiny rather than remain a pawn of external forces. Values like these, while generally implicit rather than explicit, are deeply held indeed, and I wonder how often debates about data interpretation are really proxies for disagreements concerning the meaning of life.

Perhaps the final resolution of the person-situation debate can teach us that the dichotomies that underlie the competition between these values, like the person-situation contrast itself, are false. For example, does acknowledging the influence of social conditions on life outcomes really make personal responsibility irrelevant? Is individual freedom of action really incompatible with being true to oneself? Must we choose between these core values, and continue to argue (perhaps implicitly) in favor of one at the expense of the other? If a close analysis of the data and theory related to the continuity and discontinuity of personality can lead to a clear comprehension that the answer to these and related questions really is "no," then personality psychology, and one of its core debates, will have made a useful contribution to human understanding.

\section{References}

Ahadi, S., \& Diener, E. (1989). Multiple determinants and effect size. Journal of Personality and Social Psychology, $56,398-406$.

Allport, G. W. (1937). Personality: A psychological interpretation. New York: Holt Rinehart \& Winston.

Bem, D. J. (1983). Constructing a theory of the triple typology: Some (second) thoughts on nomothetic and idiographic approaches to personality. Journal of Personality, 51, 566-577.

Bem, D. J., \& Allen, A. (1974). On predicting some of the people some of the time: The search for cross-situational consistencies in behavior. Psychological Review, 81, 506-520.

Bem, D. J., \& Funder, D. C. (1978). Predicting more of the people more of the time: Assessing the personality of situations. Psychological Review, 85, 485-501.

Chaplin, W. F. (1991). The next generation of moderator research in personality psychology. Journal of Personality, 59, 143-178.

Fleeson, W. (2001). Towards a structure- and process-integrated view of personality: Traits as density distributions of states. Journal of Personality and Social Psychology, 80, 1011-1027.

Fleeson, W. (2004). Moving personality beyond the person-situation debate: The challenge and opportunity of within-person variability. Current Directions in Psychological Science, 13, 83-87.

Funder, D. C. (2001). Personality. Annual Review of Psychology, 52, 197-221.

Funder, D. C., \& Colvin, C. R. (1991). Explorations in behavioral consistency: Properties of persons, situations and behaviors. Journal of Personality and Social Psychology, 52, 773-794.

Funder, D. C., Furr, R. M., \& Colvin, C. R. (2000). The Riverside behavioral Q-sort: A tool for the description of social behavior. Journal of Personality, 68, 450-489.

Funder, D. C., \& Ozer, D. J. (1983). Behavior as a function of the situation. Journal of Personality and Social Psychology, 44, 107-112.

Furr, R. M., \& Funder, D. C. (2004). Situational similarity and behavioral consistency: Subjective, objective, variable-centered and person-centered approaches. Journal of Research in Personality, 38, 421-447.

Kelly, H. H., Holmes, J. G., Kerr, N. L., Reis, H. T., Rusbult, C. E., \& Van Lange, P. A. M. An atlas of interpersonal situations. Cambridge University Press, Cambridge, England. 
Kenrick, D. T., \& Funder, D. C. (1988). Profiting from controversy: Lessons from the person-situation debate. American Psychologist, 43, 23-34.

McClelland, D. C. (1984). Is personality consistent. In D. McClelland (Ed.), Motives personality and society (pp. 185-211). New York: Praeger.

Mehl, M. R., \& Pennebaker, J. W. (2003). The sounds of social life: A psychometric analysis of students' daily social environments and natural conversations. Journal of Personality and Social Psychology, 84, 857-870.

Mischel, W., \& Shoda, Y. (1995). A cognitive-affective system theory of personality: Reconceptualizing situations, dispositions, dynamics, and invariance in personality structure. Psychological Review, 102, 246-268.

Lewin, K. (1951). Field theory in social science. New York: Harper.

Skinner, B. F. (1938). The behavior of organisms: An experimental analysis. New York: Macmillan.

Ten Berge, M. A., \& De Raad, B. (2002). The structure of situations from a personality perspective. European Journal of Personality, 16, 81-102.

Van Heck, G. L., Perugini, M., Caprara, G. V., \& Froeger, J. (1994). The Big Five as tendencies in situations. Personality and Individual Differences, 16, 715-731.

Wolf, H., Borkenau, P., Angleitner, A., Riemann, R., \& Spinath, F. M. (2004). Multi-method assessment of personality: An observational study of adult twins. Behavior Genetics, 34, 665.

Wright, J. C. (2001). Syndromal versus contextualized personality assessment: Differentiating environmental and dispositional determinants of boys' aggression. Journal of Personality and Social Psychology, 81, 1176-1189.

Yang, Y., Read, S.J., \& Miller, L.C. (in press). A taxonomy of situations from Chinese idioms. Journal of Research in Personality. 